
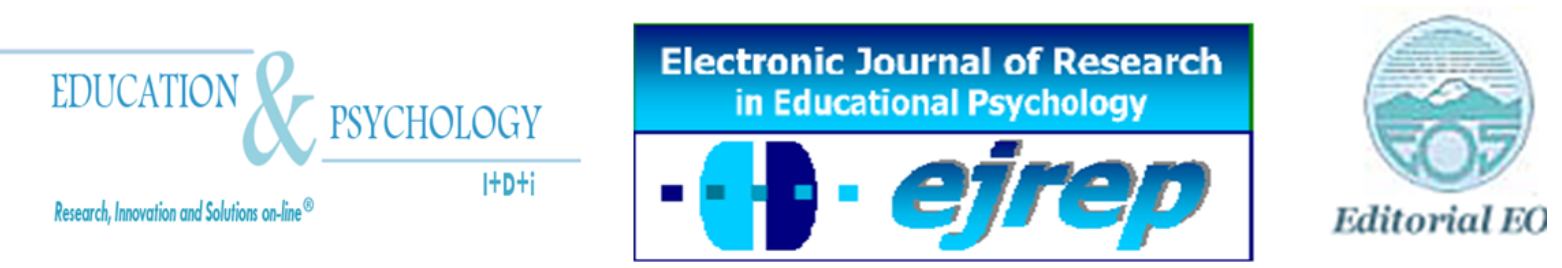

\title{
Enfoques en la investigación de la regulación de escritura académica: Estado de la cuestión
}

\section{Montserrat Castelló ${ }^{1}$, Gerardo Bañales Faz $^{2}$, Norma Alicia Vega López ${ }^{2}$}

\author{
${ }^{1}$ Facultad de Psicología, Universitat Ramon Llull, Barcelona \\ ${ }^{2}$ Unidad Académica Multidisciplinaria de Ciencias, Educación y Humanidades, \\ Universidad Autónoma de Tamaulipas, México
}

\author{
España / México
}

Correspondencia: Montserrat Catelló. C/Císter 34, 08022. Barcelona. España. E-mail: MontserratCB@blanquerna.url.edu

(C) Education \& Psychology $\mathrm{I}+\mathrm{D}+\mathrm{i}$ and Editorial EOS (Spain) 


\title{
Resumen
}

La composición efectiva de textos académicos y/o profesionales es una tarea compleja que requiere el uso de procesos de regulación. En los últimos años, estos procesos han sido estudiados preferentemente desde cuatro enfoques teóricos diferentes: cognitivo, sociocognitivo, sociocultural y socialmente compartido. En este trabajo se analizan las principales premisas teóricas y los estudios empíricos realizados desde cada enfoque y se reflexiona sobre el estado del conocimiento actual en el área de la regulación de la escritura. A modo de conclusión se delimitan aquellos problemas no resueltos que deberían orientar el futuro de la investigación.

Palabras Clave: procesos de escritura, autorregulación, co-regulación, regulación compartida, investigación de la escritura.

Recibido: 25/05/10 Aceptación Inicial: 25/05/10 Aceptación Definitiva: 08/07/10

\begin{abstract}
The effective composition of academic and/or professional texts is a complex task that needs the use of regulation processes. In the last years these processes have been studied from four research approaches: cognitive, sociocognitive, sociocultural and socially shared. In this work there are analyzed the principal theoretical premises and the empirical studies realized from each approach and there is delimited the state of the current knowledge in the field of writing regulation. As conclusion there are delimited those not solved problems that should face the future of the research.
\end{abstract}

Keywords: writing processes, self-regulation, co-regulation, shared regulation, writing research.

Received: 05/25/10 Initial Acceptance: 05/25/10 Definitive Acceptance: 07/08/10 


\section{Introducción}

Enseñar a escribir de manera efectiva es una necesidad vigente tanto en los diversos contextos educativos (Castelló, 2007) como profesionales (Quinlan y Alamargot, 2007), necesidad de la que la investigación se ha ocupado ampliamente en las últimas décadas. Una de las conclusiones que dicha investigación ha puesto de manifiesto de forma repetida, en diferentes contextos y también bajo condiciones diversas, es que esa producción efectiva de un texto ajustado a las demandas de una situación comunicativa requiere el uso de estrategias y procesos de regulación de la composición escrita (Castelló, 2009; Graham y Harris, 2000; Zimmerman y Riesemberg, 1997). La investigación acerca de las estrategias y procesos de regulación de la escritura académica en contextos de educación superior, tanto en lo que se refiere a su conceptualización como a su enseñanza, es el tema que nos ocupa en este artículo.

En los últimos treinta años, los procesos de regulación de la escritura en contextos de producción académica, han sido estudiados desde, al menos, cuatro enfoques teóricometodológicos que se diferencian por su adscripción a presupuestos preferentemente cognitivos, sociocognitivos o socioculturales; además, en los últimos años, es posible distinguir una perspectiva emergente, aunque estrechamente vinculada al enfoque sociocultural, que considera la regulación como un proceso individual a la vez que socialmente compartido, a la que dedicaremos también nuestra atención. A nivel teórico, estos enfoques entienden de forma diferencial los procesos de regulación de la escritura y, consecuentemente, en sus formulaciones otorgan diferentes roles a los factores metacognitivos, motivacionales y contextuales implicados en los mismos. A nivel metodológico, los procedimientos de recogida y análisis de datos que se enfatizan en cada caso pueden situarse dentro de un continuum que va desde aquellos que cifran su interés en el nivel individual (ej. enfoque cognitivo) hasta los que se interesan por el análisis de la interacción en situaciones de escritura colaborativa (ej. enfoque de la regulación socialmente compartida).

El objetivo de este artículo es el de revisar estos enfoques para ofrecer un análisis actualizado del estado de la cuestión sobre el estudio de los procesos de regulación en la escritura. Para ello, en primer lugar analizaremos las premisas teóricas y las opciones 
metodológicas defendidas por cada uno de ellos. En segundo lugar, revisaremos aquellos estudios empíricos sobre los procesos de regulación adscritos a cada enfoque que han hecho aportaciones relevantes al ámbito de estudio, señalando a su vez los aspectos todavía pendientes de investigación en cada caso. Como hemos avanzado, la revisión se limita especialmente a la investigación realizada en el contexto de la educación superior. En tercer lugar, realizaremos una síntesis comparativa de las nociones teóricas y aproximaciones metodológicas prototípicas de cada perspectiva o enfoque. Finalmente, a modo de conclusión, estableceremos las líneas prospectivas de la investigación en el ámbito de la regulación de la escritura académica en base a los problemas que este ámbito de investigación tiene actualmente.

\section{La regulación de la escritura desde la perspectiva cognitiva}

La mayoría de los autores coinciden en señalar la década de los ochenta y la formulación de Hayes y Flower (1980) como el despegue del estudio de los procesos de composición desde el punto de vista cognitivo y, de forma relacionada, de las primeras explicaciones relativas al control metacognitivo -regulación- de estos procesos. Desde el modelo inicialmente formulado por dichos autores, la composición de un texto es entendida como un problema a resolver a través de complejas actividades cognitivas de planificación, textualización y revisión que el escritor necesita controlar (regular) de manera recursiva y coordinada mediante un proceso de monitoreo (Hayes y Flower, 1980).

Más allá de esta formulación inicial, actualmente las teorías cognitivas postulan que la regulación o el control de la escritura -según la nomenclatura más habitualmente utilizada en sus trabajos- se realiza mediante los procesos de planificación y revisión, conceptuados como dos sistemas complejos e interactivos de control metacognitivo (ver Alamargot y Chanquoy, $2001 \mathrm{y}$ Roussey y Piolat, 2005 para una revisión detallada de las diferentes nociones acerca de la regulación o control de la escritura dentro de la perspectiva cognitiva). Desde estas teorías, la planificación es considerada como un sistema de regulación descendente -arriba-abajo (topdown)- basado en el proceso de establecimiento de objetivos (goal setting) (Roussey y Piolat, 2005). A través de este proceso el escritor activa o construye una red de objetivos y subobjetivos

(network goals) que, a modo de esquema o modelo mental, le permiten controlar y coordinar la 
totalidad de la producción textual (Flower, Schriver, Carey, Haas y Hayes, 1989; Hayes, 1996). Por su parte, la revisión es considerada un sistema de regulación ascendente -abajo-arriba (bottom-up)- que, a través de los procesos de detección, diagnóstico y reparación de problemas, permite también al escritor monitorear y controlar el logro de la red de objetivos establecida, utilizando diversas estrategias de solución (Hayes, Flower, Schriver, Stratman y Carey, 1987).

Al amparo de esta conceptualización, se han generado dos grandes líneas de investigación sobre el impacto de ambos mecanismos de control metacognitivo -planificación y revisión- en la calidad del texto. Los trabajos dedicados a la planificación, se centraron preferentemente en el análisis de los objetivos de escritura que se planteaban los escritores y su impacto de en la calidad del texto (Carey, Flower, Hayes, Schriver y Haas, 1989; Flower y Hayes, 1980; Flower et al., 1989). En un estudio pionero, Flower y Hayes (1980) analizaron los objetivos utilizados por escritores expertos y novatos (estudiantes universitarios) para escribir un texto expositivo. Los resultados señalan que los expertos dedicaron el doble de tiempo a analizar la demanda de escritura, establecieron más objetivos relacionados con el contenido y se plantearon también más objetivos retóricos dirigidos a causar un efecto en el lector o a controlar su imagen de autores en el texto de lo que lo hicieron los escritores novatos; éstos dedicaron una menor cantidad de tiempo para el análisis de la demanda y únicamente establecieron objetivos de contenido y formato del texto, sin tener en cuenta la necesidad de plantearse objetivos retóricos o de imagen del autor.

Sin embargo, en este trabajo no se mencionan los efectos que tales objetivos tuvieron en la calidad del texto, algo de lo que sí se ocuparon Carey et al. (1989). Entre sus principales resultados cabe destacar el hecho de que los mejores textos fueron los producidos por aquellos escritores que establecieron una mayor cantidad de objetivos al inicio del proceso de composición y además lo hicieron de forma cualitativamente diferente, incluyendo aspectos no sólo de contenido, audiencia y formato sino también aspectos fundamentalmente retóricos como por ejemplo la imagen del autor.

Precisando algo más la definición relativa a la calidad de los objetivos, Flower et al. (1989) distinguieron entre objetivos globales (a nivel de texto) y locales (a nivel de oración). 
Mediante el análisis de protocolos en pensamiento en voz alta, los autores concluyeron que los escritores expertos establecían objetivos de contenido (content goals) a nivel global y local de manera más integrada, lo que les permitía producir textos de mayor calidad, a diferencia de los escritores novatos que establecían objetivos de contenido sólo a nivel local, produciendo textos con cierta coherencia a nivel de los párrafos, pero desorganizados a nivel global. La investigación posterior ha precisado que el establecimiento de objetivos de contenido tanto a nivel global como local, permite a los escritores generar y recuperar las ideas de manera más jerarquizada e integrada, ayudándoles a producir textos con mayor coherencia macro y micro estructural (Dansac y Alamargot, 1999).

Por otro lado, y atendiendo al tipo de objetivos y su rol específico en los procesos de control y regulación, algunos autores han señalado el papel diferencial que tienen los objetivos de proceso (process goals) como mecanismo de regulación, frente a los de contenido (content goals) o de audiencia (audience goals) (Flower y Hayes, 1981; Hayes y Nash, 1996). Para estos autores, mientras los objetivos de contenido tienen que ver con definir lo que el escritor quiere decir y los objetivos de audiencia tienen que ver con definir el efecto que se quiere causar en el lector, los objetivos de proceso "son esencialmente las instrucciones que las personas se dan a sí mismas acerca de cómo realizar el proceso de escritura, ej...empezaré por la introducción...volveré después a esto" (Flower y Hayes, 1981, p.377). En esta misma línea, pero más recientemente Torrance y Galbraith (2006) pusieron de manifiesto que los escritores consiguen reducir las demandas de procesamiento y gestión de la información en la memoria a corto y largo plazo, con la consiguiente mejora de los procesos de regulación, cuando deciden dedicarse primero, y de manera exclusiva, a explorar y organizar las ideas a través del establecimiento de notas, esquemas visuales (outlining) o de borradores (rough draft), sin preocuparse por escribir de acuerdo a unas condiciones retóricas y de prosa formal, es decir antes de textualizar formalmente las ideas,.

La segunda línea de investigación desarrollada desde la perspectiva cognitiva, como ya hemos avanzado se ha ocupado del impacto de los mecanismos de control metacognitivo que conlleva la revisión de la calidad del texto (Flower et al., 1989; Hayes et al., 1987; Midgette, Haria y McArthur, 2008). En este sentido, ya los trabajos pioneros de Hayes et al. (1987) 
analizaron qué problemas detectaban y qué soluciones adoptaban para resolverlos los escritores expertos y novatos (estudiantes universitarios) durante la revisión de un texto expositivo que contenía problemas a nivel local (ej. ortografía) y problemas a nivel global (ej. objetivos del texto no cumplidos). Los resultados mostraron que los expertos detectaron un $42 \%$ de los problemas a nivel global a diferencia del 15\% de los novatos, de tal forma que la resolución de estos problemas globales permitió a los expertos producir textos de mayor calidad (Hayes et al., 1987). Según los autores, estos resultados indican que los expertos mostraron una mayor capacidad para realizar y utilizar estratégicamente tres tipos de diagnostico diferenciados de los problemas locales y globales en términos de intenciones, reglas y principios, y cinco tipos de soluciones de problemas denominadas: ignorar el problema (ignore), posponer el esfuerzo para resolver el problema (delay), buscar más información para resolver el problema (search), reescribir el texto mediante la elaboración de un borrador o el parafraseo (rewrite) y revisar el texto intencionalmente para corregir problemas tomando la información facilitada por el diagnostico (revising).

La investigación reciente indica que estos procesos de detección y solución de problemas que utilizan a menudo los escritores expertos pueden deberse a un mayor dominio de diferentes "modos de control" tales como el control sobre automatismos, sobre las reglas y sobre los conocimientos (Roussey y Piolat, 2005). En este sentido, en estudios de intervención recientes se confirma la importancia de un mayor dominio de estos modos de control dado que los estudiantes en situaciones en las que recibieron formación para detectar problemas en el texto a partir de objetivos de contenido (por ejemplo, agregar información) o de audiencia (por ejemplo, modificar contenido para persuadir de un argumento), produjeron ensayos argumentativos de mayor calidad, a diferencia de los estudiantes que sólo recibieron ayudas generales o que no recibieron ayudas (Midgette, Haria y McArthur, 2008).

En conjunto, los estudios sobre el establecimiento de objetivos y la detección-solución de problemas durante los procesos de planificación y revisión realizados desde la perspectiva cognitiva han sido claves para comprender mejor el impacto de los procesos de control metacognitivo en la calidad de los textos. Además, en los últimos años, y en un intento por superar las limitaciones respecto a la falta de contextualización de los resultados obtenidos desde 
esta perspectiva, algunos trabajos han abordado de forma funcional y dinámica los procesos de composición escrita (Rijlaarsdam y van den Bergh, 2006). Los resultados de éstos y otros trabajos (ver, por ejemplo, van den Bergh y Rijlaarsdam, 2007) ponen de manifiesto la multifuncionalidad que pueden tener las actividades de control metacognitivo según su activación en los diferentes momentos temporales del proceso de composición, analizando también su impacto diferencial en la calidad final de los textos (Olive y Piolat, 2003; Roussey y Piolat, 2005).

En este sentido, también Galbraith y Torrance (2004), enfatizando este carácter dinámico y recursivo de los procesos de composición, establecieron que las actividades de revisión tienen al menos dos funciones distintas dependiendo del tipo de planificación que utilicen los escritores. Así, cuando los escritores empleaban inicialmente un procedimiento de esquema (outlining), utilizaban la revisión para detectar y solucionar diversos tipos de problemas a fin de lograr unos objetivos retóricos presentes en su esquema; en cambio, cuando se utilizaba un procedimiento interactivo generando el contenido de manera exploratoria a través de la elaboración de borradores parciales del texto, los escritores utilizaban la revisión para identificar y seleccionar posibles ideas y objetivos con la finalidad de guiar la elaboración de borradores adicionales. En base a estos resultados concluyen que, dado que la mayoría de los escritores utilizan ambos procedimientos de planificación de contenido, lo que importa es que entiendan las funciones de las diferentes formas de revisión y planificación cómo ambas se relacionan con otras actividades del proceso de escritura.

A pesar de la importancia de dichas aportaciones, cabe mencionar que todavía sabemos poco acerca del papel que tienen tanto el nivel de dominio del conocimiento de tema (topic knowledge) como del genero (genre knowledge) en los procesos de regulación metacognitiva de la escritura (ver McCutchen, 2000 y Torrance y Galbraith, 2006 para una revisión del papel ambos conocimientos en los procesos de composición). Por otra parte, en los últimos años diversos autores cercanos a posiciones sociocognitivas (Zimmerman y Riesemberg, 1997; Zito, Adkins, Gavins, Harris y Graham, 2007), han señalado que además de los procesos de control metacognitivo en sí mismos, es necesario explicar y estudiar los procesos de regulación en relación a aspectos motivacionales, conductuales y contextuales que los escritores llevan a cabo 
en mayor o menor medida en toda actividad de composición escrita para entender cabalmente el impacto de la regulación en el rendimiento de escritura, aspecto del que nos ocuparemos en el apartado siguiente.

\section{La regulación de la escritura desde la perspectiva sociocognitiva}

En la década de los noventa, Zimmerman y Riesemberg (1997) difundieron una nueva conceptuación de la escritura, que conocemos con el nombre de sociocognitiva, según la cual "La escritura es más que la mera expresión escrita de una habilidad cognitiva: es un proceso social y cognitivo en donde los escritores... usan una variedad de métodos conductuales así como cognitivos para recabar y mantener experiencias afectivas y motivación" (Zimmerman y Riesemberg, 1997, p.76)

En cuanto a los procesos de autorregulación de la escritura, los mismos autores postularon que podían ser entendidos como los "pensamientos, sentimientos y acciones auto iniciadas que los escritores utilizan para lograr varios objetivos de escritura, como mejorar sus habilidades de escritura o mejorar la calidad del texto que han creado" (Zimmerman y Riesemberg, 1997, p.76).

Bajo estas premisas, en su teoría inicial Zimmerman y Riesemberg (1997) propusieron que la regulación de la escritura se lleva a cabo mediante un "sistema tríadico de procesos de autorregulación" compuesto por: procesos de regulación del ambiente, de la conducta y de la persona; No obstante, recientemente la formulación inicial de la autorregulación de la escritura como sistema tríadico ha sido reconceptualizada por los autores (Zimmerman y Kitsantas, 2007) al plantear un modelo cíclico de la autorregulación de la escritura compuesto de tres fases de regulación de la escritura: fase de premeditación (forethought phase), fase de ejecución (performance phase) y la fase de auto-reflexión (self-reflection phase). Cabe mencionar que los elementos que componen cada una de las tres fases del modelo, están basadas en la investigación sobre autorregulación en diversos ámbitos académicos, deportivos y profesionales (ver Zimmerman, 1998 y Zimmerman y Kitsantas, 2007 para una revisión detallada de dichas investigaciones). 
Desde el punto de vista metodológico, la mayoría de los estudios desarrollados bajo la perspectiva sociocognitiva se concretan en diseños de intervención basados preferentemente experimentales o cuasi-experimentales y en su mayoría con poblaciones de estudiantes de secundaria y bachillerato y en menor medida de universidad.

Destacan dos grandes líneas de investigación, orientadas a la enseñanza y aprendizaje de estrategias de escritura: la primera centrada en los estudios de intervención de Zimmerman y colaboradores en los que se puso a prueba su modelo de adquisición de habilidades de regulación (Zimmerman, 2000) y la segunda representada por Graham y Harris (2009) que también se ocupó del desarrollo y la adquisición de estrategias de auto-regulación mediante la propuesta denominada Self-Regulated Strategy Development (SRSD).

La primera de estas líneas de investigación (Zimmerman, 2000) propone que la mejor manera en que los estudiantes pueden aprender nuevas habilidades de escritura es mediante un proceso de enseñanza-aprendizaje basado en cuatro niveles secuenciales: observación, emulación, auto-control y autorregulación. En el nivel de observación, mediante el modelado, el estudiante adquiere una imagen clara de cómo deben ser ejecutadas las estrategias de regulación de la escritura. En este nivel el estudiante aprende observando las acciones y escuchando las descripciones de los modelos y la motivación proviene de los reconocimientos que el modelador ofrece al aprendiz. Por ejemplo, se puede modelar el uso del mapa de ideas como técnica para organizar los contenidos de un texto expositivo de manera jerárquica y secuencial; el estudiante en este nivel solo se limitaría a observar y atender las explicaciones sobre el uso de esta técnica.

En el nivel de emulación el estudiante aprende a regular su escritura emulando la ejecución del modelo. Se considera que el estudiante alcanza este nivel cuando puede ejecutar por sí mismo la habilidad modelada y cuando la fuente de motivación del aprendiz se basa en la mejora de su rendimiento gracias a procesos de retroalimentación conductual y social. Siguiendo con el ejemplo, en este nivel el estudiante practicaría la técnica del mapa de ideas de acuerdo a los criterios y estándares de elaboración señalados bajo la supervisión del profesor. 
En el nivel de auto-control el estudiante se dirige a mejorar y automatizar la habilidad o técnica de escritura aprendida, mediante la practica autoiniciada y autodirigida. En este nivel el aprendiz compara su nivel de ejecución con los estándares de regulación de dicha habilidad o técnica aprendida previamente del modelo y su motivación se basa en las reacciones de autosatisfacción surgidas por el cumplimiento o superación de tales estándares. Por ejemplo, el estudiante puede comparar el mapa de ideas que elabore para un nuevo tema con los mapas modelos.

Finalmente, en el nivel de autorregulación el estudiante aprende a adaptar las habilidades y técnicas aprendidas ante tareas de escritura con condiciones diferentes. En este nivel, el desafío básico del estudiante es el de transferir lo que aprendió mediante procesos modelados a situaciones diversas en las que debe anticipar los resultados que puede obtener mediante el uso de una determinada técnica. Por ejemplo, suponiendo que el estudiante hubiera aprendido no solo la técnica del mapa de ideas sino también la técnica de comparación y contraste como herramienta para organizar las ideas del texto, el desafío para el estudiante podría ser decidir qué técnica utilizar para organizar el contenido del nuevo texto en cuestión.

En resumen, Zimmerman (2000) plantea que estos cuatro niveles secuenciales permiten un desarrollo progresivo de las habilidades de regulación que pasan de estar socialmente controladas y motivadas a estar autocontroladas y automotivadas. La premisa central de este modelo es que "los niveles de observación y de emulación $\left(\begin{array}{lll}1 & y & 2\end{array}\right)$ representan experiencias de aprendizaje social que preparan a los aprendices para lograr competencia en los niveles de autocontrol y autorregulación (3 y 4)”(Zimmerman y Kitsantas, 2002, p.660).

En los últimos años, los autores han realizado diversos estudios de intervención para estudiar el impacto que tienen estos cuatro niveles de instrucción en la adquisición de diversas habilidades de escritura y en diversos factores motivacionales como por ejemplo la percepción de auto-eficacia como escritor (Zimmerman y Kitsantas, 1999; 2002). En líneas generales, sus resultados señalan la eficacia del modelo de intervención puesto que refrendan la idea del desarrollo secuencial y multinivel en la adquisición de las habilidades de regulación de la escritura y de su impacto en diversos factores motivacionales. 
Sin embargo, como los autores reconocen, estos resultados se obtuvieron en tareas de escritura altamente formalizadas y estructuradas lo que evidentemente las convierte en tareas diferentes - probablemente menos complejas- que las tareas de escritura académica en contextos de educación superior, a menudo abiertas y con múltiples posibles concreciones y de revisión más complejas.

Por su parte, la segunda de las líneas de investigación identificadas en esta perspectiva, se inicia en la década de los noventa cuando Harris y Graham (1992) dieron a conocer el modelo de "Desarrollo de Estrategias de Autorregulación" (Self-Regulated Strategy DevelopmentSRSD). En el modelo SRSD, la escritura (y su desarrollo) se entiende como un proceso complejo basado en cuatro factores: estrategias, habilidades de transcripción, conocimientos y motivación (Graham y Harris, 2009).

Las estrategias son entendidas como habilidades de autorregulación tales como el establecimiento de objetivos, el auto-monitoreo, la provisión de auto-instrucciones, la autoevaluación y el auto-reforzamiento, que permiten al escritor dirigir de manera activa y controlada el proceso de composición y cuyo uso supone una alta demanda cognitiva (high-level skills). Por habilidades de transcripción se entienden principalmente las habilidades de caligrafía (handwriting) y ortografía (spelling) que permiten al escritor transformar las palabras que quiere decir en símbolos escritos impresos en una hoja, cuyo uso supone una baja demanda cognitiva (low-level skills). El conocimiento, se refiere a los diferentes tipos de conocimientos implicados en la escritura tales como el conocimiento del tema, de la audiencia, del género, lingüístico, entre otros. Finalmente, en la motivación se da importancia a varios constructos motivacionales tales como la autoeficacia, las actitudes positivas y negativas hacia la escritura, entre otros.

El modelo SRSD asume una concepción sociocognitiva de la regulación de la escritura entendiéndola como "el proceso mediante el cual los estudiantes activan y mantienen procesos cognitivos, conductas y afectos, que son sistemáticamente orientados hacia el logro de objetivos" (Zito et al., 2007, p. 78). El modelo SRSD es un método de enseñanza directa de estrategias de regulación de la escritura, basado en 6 etapas instruccionales: 1) desarrollo y activación conocimiento previo, 2) discusión sobre el uso de las estrategias, 3) modelado 4) 
memorización, 5) apoyo y guía en su uso y 6) ejecución independiente (ver Graham y Harris, 2009 para una explicación detallada de cada una de ellas).

En los últimos 30 años Graham, Harris y colaboradores han investigado mediante diseños experimentales y cuasi-experimentales el impacto que tiene el modelo de intervención SRSD en el aprendizaje de diversas estrategias de regulación de la escritura, en las habilidades de transcripción, en los conocimientos de la escritura y en varios factores motivacionales (De la Paz y Graham, 2002; Graham, Harris y Mason, 2005; ver Graham, 2006 para una revisión exhaustiva de estudios que han analizado el impacto del modelo SRSD en el aprendizaje de la escritura). Los resultados, en líneas generales, ponen de manifiesto la efectividad del modelo SRSD para mejorar el rendimiento de escritura expositiva, especialmente de los estudiantes en niveles tempranos del desarrollo y también de los que pertenecen a colectivos con dificultades en el aprendizaje de la escritura.

Precisamente, el análisis de la enseñanza de estrategias de escritura y de las habilidades de regulación en estudiantes con dificultades de aprendizaje en el ámbito de la enseñanza primaria y secundaria (Graham, 2006; Graham, Harris y Mason; 2005), ha sido una de las líneas prioritarias de investigación de estos autores. En este colectivo de estudiantes, sus resultados muestran la potencia del modelo en, al menos, 5 aspectos del rendimiento en escritura de los estudiantes: a) mejora en el aprendizaje y uso de estrategias de regulación de la escritura tanto en la panificación como en la revisión de diferentes tipos de textos, b) aprendizaje de conocimientos de escritura básicos, c) calidad de los escritos, d) actitud hacia la escritura, e) autoeficacia. Sin embargo, también ponen de manifiesto la dificultad de medir las percepciones de autoeficacia con estudiantes de primaria y la necesidad de clarificar el papel de la ayuda entre iguales en la efectividad de la enseñanza de estrategias y conocimientos mediante el modelo SRSD.

En resumen, los modelos sociocognitivos de Zimmerman (2000) y de Graham y Harris (2009) ofrecen una explicación teórica de la regulación de la escritura que, a diferencia de los modelos cognitivos, enfatiza la importancia de varios factores cognitivos, conductuales, afectivomotivacionales y ambientales que intervienen en toda actividad de escritura y que deben ser 
regulados a fin de lograr unos determinados objetivos de escritura. En un sentido amplio, los estudios sociocognitivos muestran evidencias de la efectividad de ambos modelos instruccionales para mejorar el rendimiento de escritura de los estudiantes en una variedad de tipos de textos. No obstante, de acuerdo con la reciente investigación sobre la motivación en la escritura (ver, por ejemplo, Hidi y Boscolo, 2007 para un estado de la cuestión), otros factores no contemplados en estos modelos, como por ejemplo el interés por el tema y la tarea, también parecen influir en la regulación de la escritura (Boscolo, Favero y Borghetto, 2007). Así, poco se sabe acerca del impacto de estos modelos en el aprendizaje y uso de estrategias de regulación de la motivación durante el proceso de escritura, que son distintas de las habituales estrategias de regulación del proceso cognitivo de escritura (Wolters, 2003, p. 192). Por otra parte, como ya hemos comentado, los efectos instruccionales positivos de ambos modelos se han obtenido de forma casi exclusiva, en contextos de educación primaria y secundaria pero resulta difícil extrapolar sus resultados a los contextos universitarios caracterizados por la existencia de tareas de escritura expositiva y o argumentativas habitualmente muy complejas (Andriessen y Coirier, 1999).

A diferencia de la perspectiva sociocognitiva que estudia los procesos individuales de los escritores para regular la escritura a nivel cognitivo, motivacional, conductual y contextual y el impacto de estos procesos de regulación en la calidad de los textos, la perspectiva sociocultural se centra principalmente en explicar y estudiar los procesos de co-regulación de la escritura. En el siguiente apartado, presentamos las premisas y estudios empíricos realizados al respecto.

\section{La regulación de la escritura desde la perspectiva sociocultural}

Desde la óptica sociocultural, la composición de un texto es considerada como una práctica discursiva, dialógica y situada, desarrollada por los miembros de una comunidad en un determinado contexto social, cultural e histórico (Castelló, 2007; Prior, 2006). Los miembros principiantes de la comunidad aprenden a escribir y a regular la composición mediante su participación en prácticas discursivas autenticas y a través de la interacción social con miembros más expertos o avanzados (Englert, Mariage y Dunsmore, 2006). 
Si pensamos en los contextos escolares, los docentes (y algunos estudiantes) en su papel de miembros avanzados proveen de manera guiada y ajustada diversos andamiajes o ayudas en términos de conocimientos, procedimientos y otras herramientas culturales con la finalidad de que el estudiante en su papel de aprendiz, se apropie de ellos y los utilice de manera autónoma e independiente (Englert et al., 2006). En este sentido, profesores y estudiantes inmersos conjuntamente en la actividad de escritura, promueven una interacción discursiva que les permite construir conocimientos y compartir significados situados sobre los planes, objetivos, procesos, y decisiones importantes dentro un espacio común de regulación. Mediante estos complejos mecanismos de andamiaje e intersubjetividad los docentes ejercen una potente mediación social que permite el traspaso o transición gradual de la regulación inicialmente dirigida por el docente (teacher-directed regulation), o por otros estudiantes más avanzados, hacia la regulación de la escritura dirigida por el propio estudiante (student-directed regulation) (Hadwin, Oshige, Gress y Winne, 2010). Así pues, de acuerdo con la noción vigotskiana de Zona de Desarrollo Próximo (ZDP), los docentes, mediante los procesos de traspaso y cesión del control, ayudan al estudiante en la apropiación de conocimientos que le permiten autorregular y resolver por sí mismo tareas de escritura complejas, para la que antes se requería la regulación y la ayuda externa. El mecanismo que permite el paso del control de la regulación externa a la autorregulación es conceptualizado desde esta perspectiva como la actividad de co-regulación (McCaslin y Hickey, 2001).

Además, la actividad de co-regulación se extiende más allá de los procesos de interacción cara a cara y media la actividad individual de regulación de la escritura que realizan los estudiantes sin la presencia física de otra persona de la comunidad (McCaslin y Hickey, 2001; Pressley, 1995), de tal modo que la actividad de regulación de la escritura, aun y cuando se realice de manera solitaria en un plano intrapsicológico, está siempre mediada por el diálogo que el escritor tiene con otras voces en un espacio o plano interpsicológico y por las ayudas y herramientas culturales interiorizadas, que permiten al escritor tomar decisiones más o menos estratégicas (Castelló, 2007; Flower, 1994; Monereo, 2007; Pressley, 1995). En este diálogo, que sucede entre los planos inter e intrapsicológicos, el escritor construye un contexto de la situación comunicativa donde negocia significados entre su voz y otras voces respecto al propósito, contenido y forma de un determinado texto (Flower, 1994; Prior, 2001). En este sentido, desde la 
perspectiva sociocultural, se asume que los procesos individuales de regulación de la escritura a nivel cognitivo-afectivo son siempre situados en y mediados por el contexto social, cultural e histórico donde estos tienen lugar, y están vinculados a la identidad asumida por el escritor (Castelló, 2007; Prior, 2006).

A partir de estas premisas, se han realizado diversos estudios sobre los procesos de coregulación de la escritura. Un primer grupo de estudios, se ha dedicado al análisis de los procesos de co-regulación entre estudiantes universitarios en situaciones de planificación colaborativa de un texto (Burnett, 1994; Higgins, Flower y Petraglia, 1992). Así por ejemplo, Higgins y colaboradores (1992) indagaron el papel de las ayudas o andamiajes entre iguales en la planifcación textual en un estudio en el que 22 estudiantes de licenciatura agrupados en díadas en las que un estudiante tenía el rol de lector y el otro de escritor- tenían que planificar sus textos, utilizando cuatro tipos de ayudas (prompts): 1) propósito o puntos principales del texto, 2) audiencia, 3) convenciones textuales y 4) contenido del tema.

Los resultados permitieron identificar dos patrones de planificación colaborativa: patrón listado y patrón interactivo. En el primero, los estudiantes en el rol de lector utilizaron las ayudas ofrecidas (propósito, audiencia, etc) como listas de preguntas que hacían al estudiante en el rol de escritor, ayudándole a verbalizar sus planes, pero sin promover en ellos una reflexión sobre los mismos en ellos ni sobre el propósito retórico. En cambio, en el patrón interactivo el estudiante en el papel de lector utilizó preguntas o cuestionamientos que exigían que su compañero explicitase sus objetivos y propósitos, pensara alternativas de solución en caso de problemas y justificara la elección de tales planes. Desafortunadamente, el estudio no aporta datos respecto a la influencia de estos patrones en la calidad del texto final ni detalla las preguntas o movimientos verbales diferenciales de ambos patrones.

En un análisis posterior de los mismos datos, Burnett (1994) puntualizó que las diadas que utilizaron patrones interactivos, se caracterizaron por el uso de procesos de andamiaje basados en cuatro tipos de movimientos verbales: dar ayudas (prompts), dar información de contenido, desafiar y dirigir al escritor. Las observaciones de Burnnet (1994) señalan que los colaboradores comprometidos y exitosos se caracterizaron por un uso diversificado y frecuente 
de los cuatro movimientos verbales, y particularmente, por el uso estratégico que hacían de cada uno de ellos, dependiendo de la necesidad y el momento en que se situara la planificación. En cambio, los colaboradores inexpertos adoptaron un papel autoritario, criticando los planes de su compañero, ofreciendo la mayoría de las ideas importantes, o asumiendo como propio el texto del compañero, confundiendo su rol de colaborador.

Burnett (1994) señala que además de estos movimientos verbales, otros factores de tipo afectivo podrían haber influido en el proceder de los colaboradores comprometidos, tales como una mayor consciencia del contexto social de la tarea, una autoimagen positiva como escritores y una mayor motivación, responsabilidad y receptividad hacia la actividad de planificación colaborativa. A pesar de la importancia empírica de estos resultados para la comprensión de los procesos de andamiaje y co-regulación de la escritura, pocos trabajos han seguido analizando en el contexto universitario los movimientos verbales y factores afectivos que dan lugar a patrones interactivos o de otro tipo en situaciones de planificación colaborativa.

Un segundo grupo de estudios ha abordado el análisis de los procesos de co-regulación entre estudiantes universitarios en situaciones de revisión de la escritura (Castelló, Iñesta y Monereo, 2009; Castelló, González e Iñesta, 2010; Cho, Schunn, y Charney, 2006). Así, Castelló y colaboradores (2010), analizaron los problemas que los estudiantes de postgrado percibían y los cambios que eran capaces de realizar para solucionarlos, en situaciones de revisión colaborativa - parejas- de tres versiones sucesivas de un capítulo de su proyecto de tesis, después de haber asistido a un taller de escritura académica.

Los resultados muestran que, algunas diadas, a lo largo de las tres sesiones de revisión, se centraron predominantemente en la resolución de problemas de coherencia a nivel de oraciones y párrafos, en la mejora de la precisión de las ideas a nivel sintáctico y estructural, y en la solución de los problemas de terminología inapropiada mediante el uso de modalizadores y la inclusión de información adicional a diferencia de otras díadas, en las que los problemas no se definían de forma precisa lo que impedía su resolución y la consecuente mejora del texto. Tampoco en este caso, el estudio señala el impacto de ambos patrones en la calidad de los textos producidos por las diadas ni analiza el discurso de los estudiantes en las sesiones de revisión colaborativa. 
Al respecto, Cho y colaboradores (2006) estudiaron los tipos de comentarios que realizaron estudiantes (de licenciatura y postgrado) y profesores universitarios a los textos de otros estudiantes y analizaron la percepción de utilidad de dichos comentarios por parte de los autores de los textos. Ambos revisores evaluaron los textos conforme a 3 dimensiones: flujo de la prosa, argumentación y avance de ideas (insight). Entre los principales resultados, destaca el hecho de que los profesores en calidad de expertos hicieron un uso mayor de comentarios directivos con respecto a la dimensión de flujo de la prosa y de la argumentación. Por su parte, los estudiantes de licenciatura utilizaron en sus revisiones comentarios directivos y de alabanza en las tres dimensiones evaluadas mientras que los estudiantes de postgrado, combinaron patrones de los expertos y de los estudiantes de licenciatura. A pesar de que los estudiantes no diferenciaron los comentarios ofrecidos por expertos y por los pares respecto a su utilidad, sí lo hicieron en relación a los comentarios directivos y de alabanza.

Un tercer grupo de estudios, se ha centrado en estudiar el papel de las "ayudas procedimentales" que ofrecen los docentes para facilitar a los estudiantes la planificación y revisión de sus textos (Allal, 2008; Wallace, 1994). Así, en un estudio reciente, Allal (2008) analizó el impacto de las discusiones de toda la clase (whole-classs) en las revisiones de los estudiantes. La muestra estaba formada por tres aulas diferentes y aún cuando los tres docentes tenían la misma secuencia didáctica, la autora encontró diferencias en la manera en que éstos ayudaron a los estudiantes a elaborar el contenido, a construir una guía para elaborar la autobiografía y a revisar los textos. Así, cuando los docentes facilitaron la discusión y participación en la construcción de las categorías de la guía y ofrecieron múltiples ejemplos de pensamiento -modelado- respecto a la forma de entender la revisión, los estudiantes realizaron un mayor porcentaje de revisiones a nivel de organización del texto y semántico produciendo mejores textos.

Aun cuando la mayor parte de los estudios socioculturales revisados en el apartado anterior, se han centrado en estudiar los procesos de co-regulación entre profesores y estudiantes o entre estudiantes, recientemente resulta posible identificar un grupo incipiente de estudios centrados en explorar los procesos individuales de regulación que llevan a cabo los escritores expertos en tareas autenticas, reconociendo su naturaleza situada, dialógica y mediada 
socialmente (Iñesta, 2009; Iñesta y Castelló, 2010), que podemos clasificar como un último y cuarto grupo dentro de esta perspectiva. En este sentido, Iñesta (2009) comparó los procesos de regulación de dos escritores expertos durante la composición de un artículo científico en dos idiomas. La unidad de análisis fue en este caso, los episodios de regulación que se consideran como secuencias de acciones que los autores implementan estratégicamente con el objetivo de resolver una dificultad identificada en el proceso de composición (Iñesta y Castelló, 2010).

Los resultados indican que es posible diferenciar entre episodios de regulación explícitos e implícitos. En los primeros, los escritores regulan intencionalmente su actuación al enfrentarse de forma consciente a retos o problemas durante la escritura. En los segundos, el escritor no es capaz de identificar ni formular ningún problema específico, pese a que su actuación pone de manifiesto una intensa actividad de regulación evidenciada en los registros de su actividad escritora a través de múltiples y variadas acciones que implican eliminaciones, modificaciones reiteradas de palabras o frases, varios intentos en la producción de una frase o un párrafo, etc. Además, en ocasiones, los episodios de regulación se resuelven durante la misma sesión de trabajo (intra-sesión), pero, en otros casos, si bien en menor medida, los episodios se retoman en varias sesiones, lo que podría indicar que para algunos aspectos de la escritura se requieren procesos de regulación permanentes -abiertos- a lo largo de todo el proceso de composición.

Finalmente, algunos datos de este trabajo muestran también que los procesos de regulación explícita e implícita que emplean los escritores expertos de manera autónoma en tareas de escritura autenticas, a pesar de que se producen en situaciones de escritura aparentemente individuales, están siempre co-regulados, es decir mediados y andamiados socialmente por los criterios y normas de calidad de las comunidades de investigación a las que los autores se dirigen.

En resumen, los estudios socioculturales sobre los procesos de co-regulación de la escritura han generado importantes contribuciones a la enseñanza de la regulación de la escritura en las últimas dos décadas (ver, por ejemplo, Englert et al., 2006 para una revisión de varias propuestas de enseñanza de la escritura de orientación sociocultural), fundamentalmente centradas en la comprensión de los complejos procesos de andamiaje e intersubjetividad en los 
que se implican escritores y colaboradores cuando realizan actividades de escritura auténticas dentro de comunidades discursivas situadas social, cultural e historicamente (Burnett, 1994; Castelló, et al., 2010; Chon, et al., 2006; Iñesta, 2009). Sin embargo, todavía sabemos relativamente poco, no sólo de la utilidad de determinados andamiajes dirigidos a facilitar la adquisición de procedimientos de regulación de la escritura, sino también de su utilización por parte de profesores y estudiantes e incluso de cómo determinadas ayudas externas (por ejemplo, programas informáticos) pueden facilitar la adquisición de conocimientos declarativos, procedimentales y metacognitivos en una situación de escritura determinada (Azevedo y Hadwin, 2005). Precisamente, en los últimos años, algunas investigaciones vinculadas al estudio de los procesos de regulación socialmente compartidos (socially shared regulation) (Hadwin, et al., 2010) que toman como unidad de análisis la interacción entre la regulación individual y colectiva en situaciones de aprendizaje colaborativo pueden suponer una contribución interesante para superar algunas de las limitaciones de los estudios realizados hasta el momento. En el apartado siguiente presentamos las premisas y estudios realizados desde esta perspectiva

\section{La regulación de la escritura desde la perspectiva socialmente compartida}

Como ya hemos anunciado al inicio de este artículo, en los últimos años, es posible identificar una perspectiva emergente en el estudio de la regulación que, aún compartiendo los presupuestos básicos de las perspectivas socioculturales, entiende la regulación como el conjunto de actividades mediante las cuales, los individuos regulan tanto su actividad individual como la actividad colectiva en la que participan (Hadwin, et al., 2010). Desde esta perspectiva denominada de la regulación situada y socialmente compartida, estas actividades permiten la coconstrucción de un mutuo entendimiento de la tarea, el establecimiento compartido de objetivos y actividades de planificación, ejecución y evaluación que los estudiantes realizan como grupo para resolver conjuntamente una tarea (Hadwin, et al., 2010). En este sentido, puede afirmarse que la regulación socialmente compartida, es una regulación colectiva o comunal en la que los procesos y productos de la regulación están distribuidos entre el grupo y a la vez son responsabilidad de cada uno de los sujetos individuales que conforman dicho grupo (Jackson, McKenzie, y Hobfoll, 2000). 
La investigación realizada desde esta perspectiva, se dirige al análisis tanto de los procesos de regulación compartida que realiza un grupo como de los procesos de regulación individual que los miembros del grupo emplean para regular a otros miembros o a todo el grupo. Al igual que en los estudios socioculturales, el análisis del discurso y los rastros de información de la actuación (trace data) son las fuentes principales para analizar los procesos de regulación (ver Hadwin, et al, 2010 para una comparativa metodológica).

Estos procesos de regulación compartida se han investigado principalmente en la resolución de problemas tanto en situaciones de aprendizaje colaborativo apoyado por ordenador -Computer Supported Collaborative Leearning-CSCL- (Vauras, Iiskala, Kajamies, Kinnunen y Lehtinen, 2003), como en situaciones de aprendizaje presencial (Volet, Summers y Thurman, 2009), pero son prácticamente inexistentes los trabajos centrados exclusivamente en la escritura. En este sentido, puede ser conveniente establecer canales de dialogo teórico y metodológico entre estos estudios y los que se ocupan del análisis de la regulación de la escritura colaborativa tanto en contextos presenciales (Lowry, Curtis y Lowry, 2004) como apoyados por ordenador (Erkens, Jaspers, Prangsma y Kanselaar, 2005). Particularmente, algunas de las nuevas plataformas diseñadas para favorecer la escritura colaborativa apoyada por ordenador (Clark, Sampson,Weinberger y Erkens, 2007), pueden permitir analizar los procesos de regulación compartida que llevan a cabo los estudiantes cuando escriben de forma colaborativa examinando la división de roles, el uso de herramientas, las emociones y conocimientos que comparten y los patrones de interacción discursivos que emplean para planificar, escribir y revisar un texto dentro de un espacio virtual socialmente compartido. Sin duda, en el futuro inmediato, el vinculo entre ambos campos puede ser un importante terreno de investigación para explorar las complejas relaciones entre la regulación individual y compartida en la producción colectiva de textos auténticos.

\section{A modo de síntesis y conclusión}

Los procesos de regulación de la composición escrita han sido estudiados en las últimas décadas desde, al menos, cuatro grandes enfoques teórico-metodológicos: cognitivo, sociocognitivo, sociocultural y social compartido (ver Tabla 1 para una síntesis comparativa). La 
perspectiva cognitiva se ha ocupado principalmente del estudio de los procesos de control metacognitivo tanto en lo que concierne al proceso de planificación -más concretamente en el establecimiento de objetivos- como al proceso de revisión y más concretamente a las actividades de detección y solución de problemas textuales. Los diseños utilizados desde esta perspectiva tienden a diferenciar la actuación de los escritores expertos en relación a la de los novatos y plantean la escritura fundamentalmente como una actividad individual. Los textos acostumbran a responder a demandas específicas, cerradas y desarrollarse durante espacios cortos y prefijados de tiempo. Desde el punto de vista metodológico, utilizan de forma preferente el análisis de protocolos de pensamiento en voz alta y diseños correlacionales y experimentales.

Por su parte, la investigación desarrollada desde la perspectiva sociocognitiva, debido, en parte, a su orientación hacia el análisis de los contextos de práctica, se ha dedicado fundamentalmente a discernir el impacto de la enseñanza de diversos procedimientos de autorregulación. Estos procedimientos se organizan en modelos que implican tanto estrategias de regulación cognitiva como afectivo-actitudinal. Los diseños adoptados desde esta perspectiva, fundamentalmente cuasi-experimentales, implican el análisis de determinadas intervenciones en el rendimiento de escritura de los estudiantes en diferentes contextos. Si bien en ocasiones las intervenciones se producen en contextos naturales, es frecuente que dichas intervenciones se realicen de forma controlada de acuerdo a las exigencias metodológicas del control de variables.

Por otro lado, los estudios realizados desde perspectivas socioculturales se han desarrollado preferentemente en contextos educativos naturales, con el objetivo de estudiar cómo se interiorizan las ayudas o andamiajes tanto de los docentes como de otros compañeros en calidad de escritores más avanzados; es decir, cómo se facilita la apropiación de la actividad de regulación de la composición escrita mediante el estudio de los procesos de co-regulación. A nivel metodológico, el diseño más habitual es el de estudios de casos, tanto individuales como múltiples, mientras que los datos analizados provienen del discurso en situaciones interactivas, registrados normalmente, a través de técnicas de registro de video, audio y/o capturas de pantalla de ordenador y complementados con la información proporcionada por diarios, autoreportes y documentos. 
Tabla 1. Síntesis comparativa de los enfoques de investigación de los procesos de regulación de la escritura

\begin{tabular}{|c|c|c|c|c|}
\hline & Cognitiva & Sociocognitiva & Sociocultural & Socialmente compartida \\
\hline $\begin{array}{l}\text { Noción de } \\
\text { escritura }\end{array}$ & $\begin{array}{l}\text { Procesos cognitivos de planeación, } \\
\text { textualización y revisión }\end{array}$ & $\begin{array}{l}\text { Actividad cognitiva, motivacional y conductual } \\
\text { compleja en interacción con } \\
\text { el ambiente social y físico }\end{array}$ & $\begin{array}{l}\text { Actividad discursiva del escritor mediada, } \\
\text { dialógica y situada dentro de una comunidad } \\
\text { discursiva y ubicada en un contexto social, } \\
\text { histórico y cultural determinado }\end{array}$ & $\begin{array}{l}\text { Actividad discursiva compartida entre miembros de } \\
\text { una comunidad discursiva y ubicada en un contexto } \\
\text { social, histórico y cultural determinado }\end{array}$ \\
\hline $\begin{array}{l}\text { Noción de } \\
\text { regulación }\end{array}$ & $\begin{array}{l}\text { Sistemas metacognitivos que utiliza } \\
\text { el escritor para controlar la } \\
\text { producción textual: } \\
\text { - Planeación: } \\
\text { establecimiento de } \\
\text { objetivos } \\
\text { Revisión: detección, } \\
\text { diagnostico y solución de } \\
\text { problemas }\end{array}$ & $\begin{array}{l}\text { Pensamientos, sentimientos y acciones auto } \\
\text { iniciadas que los escritores utilizan para lograr } \\
\text { varios objetivos de escritura, como mejorar sus } \\
\text { habilidades de escritura o mejorar la calidad del } \\
\text { texto que han creado }\end{array}$ & $\begin{array}{l}\text { Proceso de traspaso de la regulación externa } \\
\text { (experto) a la autorregulación interna } \\
\text { (aprendiz) de los conocimientos que permiten } \\
\text { la regulación de una determinada actividad } \\
\text { discursiva. } \\
\text { Proceso de autorregulación co-regulados } \\
\text { socialmente, a partir de la interiorización y } \\
\text { uso situado de diversas ayudas e herramientas } \\
\text { culturales durante la realización de una } \\
\text { determinada actividad discursiva. }\end{array}$ & $\begin{array}{l}\text { Procesos mediante los que múltiples personas regulan } \\
\text { una actividad discursiva colaborativa } \\
\text { Procesos de co-construcción de un mutuo } \\
\text { entendimiento de la tarea, el establecimiento } \\
\text { compartido de objetivos y las actividades de } \\
\text { planeación, ejecución y evaluación que los estudiantes } \\
\text { realizan como grupo para resolver conjuntamente una } \\
\text { tarea de escritura colaborativa }\end{array}$ \\
\hline $\begin{array}{l}\text { Unidad de } \\
\text { análisis }\end{array}$ & $\begin{array}{l}\text { Procesos metacognitivos de } \\
\text { planeación y revisión }\end{array}$ & $\begin{array}{l}\text { Procesos de auto-regulación de las actividades } \\
\text { cognitivas, factores motivacionales, conductas } \\
\text { y ambiente implicadas en la producción textual }\end{array}$ & $\begin{array}{l}\text { Procesos de co-regulación presenciales entre } \\
\text { pares en tareas de planeación o revisión } \\
\text { reciproca de un texto individual } \\
\text { Procesos de co-regulación entre profesor y } \\
\text { estudiantes a nivel de toda la clase (whole- } \\
\text { class) o de tutoría } \\
\text { Procesos individuales de autorregulación co- } \\
\text { regulados socialmente a través de diversos } \\
\text { medios mediadores (ej. dialogo con otras } \\
\text { voces, condiciones de producción textual, } \\
\text { etc) }\end{array}$ & $\begin{array}{l}\text { Procesos de regulación compartida que realiza un } \\
\text { grupo, y los procesos de regulación individual que los } \\
\text { miembros del grupo emplean para regular a otros } \\
\text { miembros o a todo el grupo durante una tarea de } \\
\text { escritura colaborativa autentica } \\
\text { Examina las contribuciones, roles, la evolución de las } \\
\text { ideas y la manera en que los grupos colectivamente } \\
\text { establecen objetivos, monitorean, evalúan y regulan su } \\
\text { espacio socialmente compartido durante una tarea de } \\
\text { escritura colaborativa autentica }\end{array}$ \\
\hline $\begin{array}{l}\text { Métodos de } \\
\text { recogida }\end{array}$ & Protocolos & $\begin{array}{l}\text { Protocolos ; Auto-reportes } \\
\text { Entrevistas: Cuestionarios }\end{array}$ & $\begin{array}{l}\text { Entrevistas : Diarios } \\
\text { Diálogos ; } \\
\text { Registros video-audio-pantalla (Ej. Camtasia) } \\
\text { Documentos (ej. borradores) }\end{array}$ & $\begin{array}{l}\text { Registro informáticos de actividad compartida e } \\
\text { individual } \\
\text { Auto-reportes }\end{array}$ \\
\hline $\begin{array}{l}\text { Métodos de } \\
\text { análisis }\end{array}$ & $\begin{array}{l}\text { Correlaciones } \\
\text { Experimentales }\end{array}$ & Estudios Cuasi-experimentales & $\begin{array}{l}\text { Estudios de caso } \\
\text { Investigaciones de aula } \\
\text { Estudios de intervención } \\
\text { Análisis del discurso } \\
\text { Análisis del contenido }\end{array}$ & $\begin{array}{l}\text { Estudios de caso } \\
\text { Análisis de datos de rastro (trace data) } \\
\text { Análisis del discurso } \\
\text { Análisis del contenido }\end{array}$ \\
\hline
\end{tabular}


Por último, cabe señalar que los estudios de regulación compartida, a pesar de su cercanía teórica con los estudios socioculturales, a nivel metodológico se distinguen por su interés en el estudio de los procesos mediante los que múltiples personas regulan una actividad discursiva colaborativa. En este sentido, analizan los procesos de regulación conjunta que los estudiantes realizan como grupo para resolver tareas de escritura colaborativa, normalmente apoyada por ordenador. Para ello, a nivel metodológico diseñan generalmente estudios de casos, recogen los datos de las actividades colectivas mediante los registros de actividad informatizados (trace data), además del resto de procedimientos mencionados en los estudios realizados desde la perspectiva sociocultural con la que también comparten los sistemas de análisis, aunque en este tipo de trabajos se intenta reducir la información de tal manera que sea posible establecer relaciones y patrones tanto en la actividad de regulación individual como en la colectiva y sus relaciones.

Para concluir queremos señalar dos desafíos actuales relacionados con la investigación sobre los procesos de regulación de la composición escrita que nos parece que se desprenden de la revisión efectuada. El primero es de tipo teórico y tiene que ver con la importancia de realizar un esfuerzo de integración de los conceptos y premisas establecidos por las distintas perspectivas que, a su vez, tenga en cuenta los actuales debates y los recientes avances teórico- conceptuales en algunas variables que se han identificado como claves en el estudio de la regulación, particularmente en el ámbito de los estudios sobre la motivación en la escritura (Hidi y Boscolo, 2007). En segundo lugar, a nivel empírico parece conveniente avanzar hacia el uso de medidas de análisis de la regulación más molares que además incluyan la dimensión temporal (Castelló, 2007; van der Bergh, y Rijlaarsdam, 2007), en el sentido de lo que implican por ejemplo los episodios de regulación propuestos por Iñesta (2009) que permiten estudiar los procesos de regulación de una manera más multifuncional, dinámica e integrada, preferiblemente en tareas de escritura autenticas. Para afrontar este desafío, en las futuras investigaciones sin duda será imprescindible el uso combinado de instrumentos de evaluación online y offline de los procesos de regulación implicados en la composición escrita (ver Fidalgo y Nicasio, 2009 para una revisión más amplia). Atender ambos desafíos nos ayudará a avanzar tanto en los desarrollos teóricos como en los diseños de investigación y en el afianzamiento de determinadas prácticas educativas en el ámbito de la escritura académica. 


\section{Referencias}

Alamargot, D. y Chanquoy, L. (2001). Through the models of writing. Dordrecht: Kluwer Academic Publishers.

Allal, L. (2008, November). The co-regulation of writing activities in the classroom. Paper presented at the International Conference « de la France au Québec : $1^{\text {ce }}$ Ecriture dans tous ses états $\gg$, Poitiers, France.

Andriessen, J. y Coirier, P. (1999). Foundations of argumentative text processing. Amsterdam: Amsterdam University Press.

Azevedo, R. y Hadwin, A. (2005). Scaffolding self-regulated learning and metacognition Implications for the design of computer-based scaffolds. Instructional Science, 33, 367379.

Boscolo, P., Favero, L. y Borghetto, M. (2007). Writing on An Interesting Topic: Does Writing Foster Interest? En S. Hidi y P. Boscolo (Eds.), Writing and Motivation (pp. 73-92). Amsterdam: Elsevier.

Burnett, R. (1994). Interactions of Engaged Supporters. En L. Flower y otros (Eds.), Making Thinking Visible: Writing, Collaborative Planning and Classroom Inquiry (pp. 67-82). Urbana, Illinois: NCTE.

Carey, L., Flower, L., Hayes, J. R., Schriver, K. A. y Haas, C. (1989). Differences in Writers' Initial Task Representations. Recuperado el 7 de febrero de 2010, del sitio web del National Writing Project: http://www.nwp.org/cs/public/print/nwpr/621

Castelló, M. (2007a). El proceso de composición de textos académicos. En M. Castello, A. Iñesta, M. Miras, I. Sole, A. Teberosky y M. Zannoto (Eds.), Escribir y comunicarse en contextos científicos y académicos. Conocimientos y estrategias (pp. 47-82). Barcelona:Grao

Castelló, M. (2009). Aprender a escribir textos académicos ¿Copistas, escribas, compiladores o escritores? En J.I. Pozo y M. del Puy Pérez (Eds.), Psicología del aprendizaje universitario: La formación en competencias (pp.120-133). Madrid: Morata.

Castelló, M. González, L. \& Iñesta, A. (2010). La regulación de la escritura académica en el doctorado: El impacto de la revisión colaborativa en los textos. Revista Española de Pedagogía, 247, 521-537 
Castelló, M., Iñesta, A. y Monereo, C. (2009). Towards self-regulated academic writing: an exploratory study with graduate students in a situated learning environment. Electronic Journal of Research in Educational Psychology, 9(3), 1107-1130

Cho, K., Schunn, C. y Charney, D. (2006). Commenting on Writing:Typology and Perceived Helpfulness of Comments from Novice Peer Reviewers and Subject Matter Experts. Written Communication, 23(3), 260-294.

Clark, D. B., Sampson, V., Weinberger, A., \& Erkens, G. (2007). Analytic Frameworks for Assessing Dialogic Argumentation in Online Learning Environments. Educational Psychology Review, 19(3), 343-374.

Dansac, C. y Alamargot, D. (1999). Accessing referential information during text composition: when and why?. En M. Torrance y D. Galbraith (Eds.), Knowing What to Write: Conceptual Processes in Text Production (pp. 76-97.). Amsterdam: University Press.

De La Paz, S y Graham, S. (2002). Explicitly Teaching Strategies, Skills, and Knowledge: Writing Instruction in Middle School Classrooms. Journal of Educational Psychology, 94(4), 687-698.

Englert, C. S., Mariage, T. y Dunsmore, T. (2006). Tenets of sociocultural theory in writing instruction research. In A. MacArthur, S. Graham \& J. Fitzgerald (Eds.), Handbook of writing research (pp. 208-221). New York: Guilford.

Erkens, G., Jaspers, J., Prangsma, M. y Kanselaar, G. (2005). Coordination processes in computer supported collaborative writing. Computers in Human Behavior, 21(3), 463-486.

Fidalgo, R. y Nicasio, J. (2009). La evaluación de la metacognición en la composición escrita. Estudios de psicología, 30(1), 51-72

Flower, L. (1994). The construction of negotiated meaning. A Social Cognitive Theory of Writing. Southern Illinois: University Press.

Flower, L. y Hayes, J. R. (1980). The Cognition of Discovery: Defining a Rethorical Problem. College Composition and Communication, 31(1), 21-32

Flower, L. y Hayes, J. R. (1981). A Cognitive Process Theory of Writing. College Composition and Communication, 32(4), 365-387.

Flower, L., Schriver, K., Carey, L., Haas, C. y Hayes, J. (1989). Planning in Writing: The cognition of a Constructive Process. Recuperado el 7 de febrero de 2010, del sitio web del National Writing Project: http://www.nwp.org/cs/public/print/resource/620 
Galbraith, D. y Torrance, M. (2004). Revision in the context of multiple drafting strategies. En L. Allal, L. Chanquoy y P. Largy (Eds.), Revisión: Cognitive and Instructional Processes (pp. 63-86). Dordrecht: Kluwer Academic Publishers.

Graham, S. (2006). Strategy instruction and the teaching of writing: A metanalysis. En C. McArthur, S. Graham y J. Fitzgerald (Eds.), Handbook of writing research (pp. 28-40). New York: Guilford.

Graham, S. y Harris, K. R. (2000). The Role of Self-Regulation and Transcription Skills in Writing and Writing Development. Educational Psychologist, 35(1), 3-12.

Graham, S. y Harris, K. R. (2009). Almost 30 Years of Writing Research: Making Sense of It All with "The Wrath of Khan". Learning Disabilities Research \& Practice, 24(2), 58-68.

Graham, S., Harris, K. R. y Mason, L. (2005). Improving the writing performance, knowledge, and self-efficacy of struggling young writers: The effects of self-regulated strategy development. Contemporary Educational Psychology, 30(2), 207-241.

Hadwin, A., Oshige, M., Gress, C. y Winne, P. (2010). Innovative ways for using gStudy to orchestrate and research social aspects of self-regulated learning. Computers in Human Behavior, 26(5), 794-805

Harris, K. y Graham, S. (1992). Helping young writers master the craft: Strategy instruction and self-regulation in the writing process. Cambridge, MA: Brookline Books.

Hayes, J. R. (1996). A new framework fro understanding cognition and affect in writing. En M. C. Levy y S. Ransdell (Eds.), The science of Writing. Theories, Methods, Individual Differences and publications (pp. 1-27). New Yersey: Lawrence Erbaum Associates.

Hayes, J. y Flower, L. (1980). Identifying the organization of writing processes. En L. W. Greeg y E. R. Steinberg (Eds.), Cognitive processes in writing (pp. 3-30). Hillsdale, New Jersey: Lawrence Erlbaum Associates, Publishers.

Hayes, J., Flower, L., Schriver, K., Stratman, J. y Carey, L. (1987). Cognitive processes in revision. En S. Rosenberg (Ed.), Advances in applied in psycholinguistics. Vol 2: Reading, writing and language (pp. 176-240). Cambridge: Cambridge University Press.

Hayes, J. y Nash, J. (1996). On the nature of planning in writing. En M. C. Levy y S. Ransdell (Eds.), The science of Writing. Theories, Methods, Individual Differences and publications (pp. 29-55). Mahwah, NJ: Lawrence Erbaum Associates. 
Hidi, S. y Boscolo, P. (2007). The multiples meanings of motivation to write. En S. Hidi y P. Boscolo (Eds.), Writing and Motivation (pp. 1-15). Amsterdam: Elsevier.

Higgins, L., Flower, L. y Petraglia, J. (1992). Planning Text Together: The Role of Critical Reflection in Student Collaboration. Recuperado el 7 de febrero de 2010, del sitio web del National Writing Project: http://www.nwp.org/cs/public/print/resource/673

Iñesta, A. (2009). The regulation of research article writing. Strategies of experts writers in spanish as their first language and in english as an international language. Tesis doctoral no publicada. Universidad Ramon Llull, Barcelona, España

Iñesta, A. y Castelló, M. (2010). From writing process to writing regulation research: the Regulation Episode as a meaningful writing strategy pattern. Learning \& Instruction (submitted)

Jackson, T., McKenzie, J. y Hobfoll, S. E. (2000). Communal aspects of self-regulation. En M. Boekaerts, P. R. Pintrich y M. Zeidner (Eds.), Handbook of self-regulation (pp. 275-300 ). San Diego: Academic Press.

Lowry, P., Curtis, A. y Lowry, M. (2004). Building a Taxonomy and Nomenclature of Collaborative Writing to Improve Interdisciplinary Research and Practice. Journal of Business Communication, 41(1), 66-99.

McCaslin, M. y Hickey, D. T. (2001). Self-regulated learning and academic achievement: A Vygotskian view. En B. J. Zimmerman y D. Schunk (Eds.), Self-regulated learning and academic achievement: Theory, research, and practice, 2nd ed., (227-252). Mahwah, NJ: Erlbaum.

McCutchen, D. (2000). Knowledge, Processing, and Working Memory: Implications for a Theory of Writing. Educational Psychologist, 35(1), 13 - 23.

Midgette, E., Haria, P., y McArthur, C. (2008). The Effects of Content and Audience Awareness Goals for Revision on the Persuasive Essays of Fifth- and Eighth-Grade Students. Reading and Writing: An Interdisciplinary Journal, 21(1), 131-151

Monereo, C. (2007). Hacia un paradigma del aprendizaje estratégico: el papel de la mediación social, del self y de las emociones. Electronic Journal of Research in Educational Psychology, 5(3), 497-534.

Olive, T. y Piolat, A. (2003). Activation des processus rédactionnels et qualité des textes. Le Langage et l' Homme, XXXVIII(2), 191-206. 
Pressley. (1995). More about the development of self-regulation: Complex, long-term, and thoroughly social. Educational Psychologist, 30(4), 207-212.

Prior, P. (2001). Voices in text, mind, and society. Sociohistoric accounts of discourse acquisition and use. Journal of Second Language Writing, 10, 55-81.

Prior, P. (2006). A sociocultural theory of Writing. En C. A. McArthur, S. Graham, y J. Fitzgerald (Eds.), Handbook of Writing Research (pp. 54-65). New York: The Guilford Press.

Quinlan, T. y Alamargot, D. (2007). Highly Effective Writers and the Role of Reading: A Cognitive Approach to Composing in Professional Contexts. En D. Alamargot y P. Terrier (Eds.), Writing documents in the Workplace (pp. 61-74). Amsterdam: Elsevier.

Rijlaarsdam, G. y van den Bergh, H. (2006). Writing process theory. A functional dynamic approach. En C. A. McArthur, S. Graham y F. Fitzgerald (Eds.), Handbook of Writing Research (pp. 41-53). New York: The Guilford Press.

Roussey, J. Y. y Piolat, A. (2005). La révision du texte : une activité de contrôle et de réflexion. Psychologie Française, 50(3), 351-372.

Torrance, M. y Galbraith, D. (2006). The processing demands of writing. En C. MacArthur, S. Graham y J. Fitzgerald (Eds.), Handbook of writing research (pp. 67-80). New York: Guilford.

van den Bergh, H. y Rijlaarsdam, G. (2007). The dynamics of idea generation during writing: An online study. En M. Torrance, L. Van Waes y D. Galbraith (Eds.), Writing and Cognition (pp.125-150). Amsterdam: Elsevier.

Vauras, M., Iiskala, T., Kajamies, A., Kinnunen, R. y Lehtinen, E. (2003). Shared regulation and motivation of collaborating peers: A case analysis. Psychologia, 46(1), 19-37.

Volet, S., Summers, M., y Thurman, J. (2009). High-level co-regulation in collaborative learning: How does it emerge and how is it sustained? Learning and Instruction, 19, 128-143.

Wallace, D. (1994). Teaching Collaborative Planning: Creating a Social Context for Writing. En L. Flower y otros (Eds.), Making Thinking Visible: Writing, Collaborative Planning and Classroom Inquiry (pp. 48-66). Urbana, Illinois: NCTE.

Wolters, C.A. (2003). Regulation of Motivation: Evaluating an Underemphasized Aspect of SelfRegulated Learning. Educational Psychologist, 38(4), 189-205. 
Zimmerman, B. J. (1998). Academic Studying and the Development of Person Skill: A SelfRegulatory Perspective. Educational Psychologist, 33(2), 73-86.

Zimmerman, B. J. (2000). Attainment of self-regulation: A social cognitive perspective. En M. Boekaerts, P. Pintrich y M. Zeidner (Eds.), Handbook of self-regulation, research, and applications (pp. 13-39). Orlando, FL: Academic Press.

Zimmerman, B. J. y Kitsantas, A. (1999). Acquiring Writing Revision Skill: Shifting From Process to Outcome Self-Regulatory Goals. Journal of Educational Psychology, 91(2), 241-250.

Zimmerman, B. J. y Kitsantas, A. (2002). Acquiring writing revision and self-regulatory skill through observation and emulation. Journal of Educational Psychology, 94(4), 660-668.

Zimmerman, B. J. y Kitsantas, A. (2007). A writer's discipline: the development of a selfregulatory skill. En S. Hidi y P. Boscolo (Eds.), Wriitng and Motivation (pp. 51-69). Amsterdam: Elsevier.

Zimmerman, B. J. y Risemberg, R. (1997). Becoming a Self-Regulated Writer: A social Cognitive Perspective. Contemporary Educational Psychology, 22, 73-101.

Zito, J. R., Adkins, M., Gavins, M., Harris, K. R. y Graham, S. (2007). Self-Regulated Strategy Development: Relationship to the Social-Cognitive Perspective and the Development of Self-Regulation. Reading \& Writing Quarterly, 23(1), 77-95. 